\title{
Specifics of Shannon Entropy Transferring Between Solar Magnetic Modes
}

\author{
Mikhaylutsa V.P.
}

\begin{abstract}
Based on Shannon entropy transfer estimation technique and Stanford's solar global magnetic field harmonic coefficients, several new facts about solar magnetic modes have been found. The entropy transferring between most of modes has been subjected to steady modulation with period near 72 solar rotations (5.38 years). As rule, amplitudes of entropy transferring modulation were less or equal $0.1 \mathrm{bit} / \mathrm{solar}$ rotation. These modulations had no relations with intensity or configuration of the solar magnetic fields. All solar magnetic modes can be divided into three different groups, which are entropy sources, entropy transmitters, and entropy targets. Thus: even zonal modes $(l ; m):(8 ; 0),(6 ; 0),(4 ; 0)$ are sources of Shannon's entropy. The group of Shannon's entropy transmitters, mainly, consists of modes having sectors $(m>0)$. Two zonal odd modes $(1 ; 0)$ and $(3 ; 0)$ are Shannon's entropy targets. It has been shown that the most of medium and small-scale solar magnetic modes (order $l>3$; degree $m>0$ ) are interdependent. The features and physical sense of these dependencies have been analyzed. In result, the conclusion has been made that an unknown process should take place in the Sun, which controls the cluster of dependent magnetic modes in accordance with some scenario. Such scenario have been revealed by means of studying the special distributions of the global surface magnetic fields. In according to the scenario the tesseral-quadrupole $(l=2 ; m=1)$ polarity distributions have been appeared periodically. The periodicity ( $\mathrm{T} \sim 72$ solar rotations) was synchronized with the phases of modulation of the Shannon entropy transfer from the tesseral-quadrupole mode. The rotation rate of the tesseral-quadrupole polarity patterns is close to Bartels rotation rate.
\end{abstract}

\section{Introduction}

The detection of change in magnitude of directional (causal) coupling between non-linear time series of solar activity values is a common subject of interest in solar physics. Understanding causal dependencies may help to simplify descriptions of complex physical processes because it constrains the coupling functions between the dynamical variables. It is particularly necessary to find such organization of observational data-sets which correspond to causal relationships in order to reveal variables that drive the dynamics.

In our investigation we have tried to reveal dependencies between solar magnetic modes. Such modes have been characterized by their energy density, which can be derived as a sum of squares of global field harmonic coefficients, g's and h's, which described the mode in spherical presentation of solar magnetic field distribution in current-free approximation, Mikhailutsa (1994):

$$
\mathrm{E}_{l m}=\frac{l+1}{4 l+2}\left(\mathrm{~g} l \mathrm{~m}^{2}+\mathrm{h} l \mathrm{~m}^{2}\right)
$$

Indexes $l$ and $m$ correspondingly are the "order" of mode (marks the number of dipoles which construct the mode) and the "degree" of mode (the number of mode's bipolarity sectors which would be on the solar sphere). Values of mode energy density are invariant for any coordinate system on the solar surface.

Below we will denote a particular magnetic mode as $(l ; m)$, for example-mode $(5 ; 2)$. 
In this study we have used Wilcox Solar Observatory data which have been obtained via the web site http://wso.stanford.edu courtesy of J.T. Hoeksema. So, our investigation of dependencies has been made for 54 solar magnetic modes, from $(1 ; 0)$ through $(9 ; 9)$. Data of magnetic mode energy densities have been organized into individual time-series covering the period of four solar cycles (564 solar rotations).

\section{Shannon's Entropy Transferring}

Schreiber (2000) has developed a promising measure of the coupling strength between two time-series - transfer entropy. Transfer entropy (TE) quantifies the amount of information transfer from one variable to the other. In a data stream of two variables, e.g., $X(t)=\left\{x_{1}, x_{2}, \ldots, x_{\mathrm{N}}\right\}$ and $Y(t)=$ $\left\{y_{1}, y_{2}, \ldots, y_{N}\right), \mathrm{TE}(X \rightarrow Y)$ from time-series $X(t)$ to $Y(t)$ is the information shared between $X^{\prime}$ s past and $Y^{\prime}$ s present, given knowledge of $Y$ 's past. Let index $i$ indicates a given point in time-series, then:

$$
\mathrm{TEX}_{\rightarrow}=\sum_{y_{i}, y_{i-1}, x_{i-1}} \mathrm{P}\left(y_{i}, y_{i-1,} x_{i-1}\right) \times \log _{2}\left[\mathrm{P}\left(y_{i} \mid y_{i-1,} x_{i-1}\right) / \mathrm{P}\left(y_{i} \mid y_{i-1}\right)\right],
$$

For pure Markov systems: $\mathrm{TEx}_{\rightarrow}=0$, because conditional probabilities in (2) are equal: $\mathrm{P}\left(y_{i} \mid y_{i-1}, x_{i-1}\right)=\mathrm{P}\left(y_{i} \mid y_{i-1}\right)$. Intuitively, $\mathrm{TE}(X \rightarrow Y)$ estimates how much better one predicts $y_{i}$ using both $x_{i-1}$ and $y_{i-1}$ over using $y_{i-1}$ alone. A nonzero value of the TE for long time-series $(N \rightarrow \infty)$ certainly implies a kind of influence of $X(t)$ on $Y(t)$. Also TE can be described as conditional mutual information, with the history of the influenced variable in the condition. Generally, it gives quantitative estimation the difference of investigated dynamic system from the similar Markov's one. Due to the universal nature of this TE technique, in each specific case of its use with real systems, the physical meaning of the results found must be carefully studied and analyzed.

It should be noted here that some critical remarks have been made about understanding of "influence", James et al. (2016). They are: Is this influence necessarily via information flow? And: Is influence necessarily direct? In short, the answer has explained that the transfer entropy (for real non-infinitive time-series) might both overestimate information flow or underestimate influence. This should be kept in mind if appropriate results are discussed. Nevertheless, such potential "overestimation" or "underestimation" cannot destroy existed or create fake dependencies if same dependencies appear on base of different time-series.

In Figure 1, there is illustration of TE between concrete cells of time-series. Entropy transfer difference values (Net TE) have been added to our investigation additionally. Net TE flux can be received if two entropy streams (direct and back) between cells are compared. Because of each cell's state relates to certain time-forward and back entropy flows must correspond to same time and cells.

We have used appropriate Shannon entropy transferring estimation software based on "Partition" method, which has been described and entirely explained in Lee et al. (2012). It has been chosen after taking into account precision, computational time and the difficulty associated with parameter selection for probability calculations. 


\section{Transfer Entropy $\operatorname{TE}\left(\mathbf{X}_{\mathrm{i}} \rightarrow \mathbf{Y}_{\mathrm{j}}\right)$}

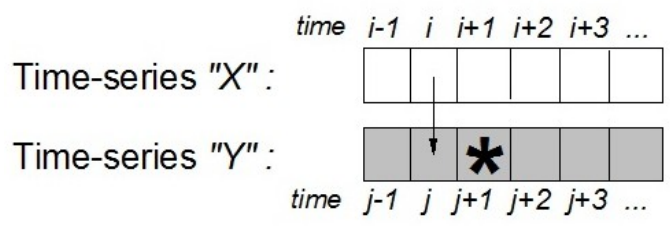

Asterisk shows predicted cell in time-series for calculating TE.

Arrow shows direction of transfer Shannon entropy between cells of time-series.

$$
\text { Net entropy flux }=T E\left(X_{i} \rightarrow Y_{i}\right)-T E\left(Y_{i} \rightarrow X_{i}\right)
$$

Figure 1. Transfer entropy between time-series of physical values. When cell's indexes are equal $(i=$ $j$ ) it is possible to estimate net entropy flux. During our calculations of TE indexes have been drifted along time-series.

\section{Main Results}

Below we present the main results of use Shannon entropy transfer technique to solar magnetic mode time-series. It was found that Shannon entropy transferring process between most of mode energy time-series has been subjected to steady harmonic modulation with period of about 72 solar rotations ( $\approx 5.38$ years). These modulations have occurred independently from intensity and phase of solar magnetic energy cycles. In the corresponding Fourier spectra of TE processes, the period of about 72 solar rotations was the only one that reached a power peak above the level of three dispersion. In the processes of information entropy transmission with modulation of its amplitude, the mode $(2 ; 1)$ was the main one. In Figure 2, this result is shown.

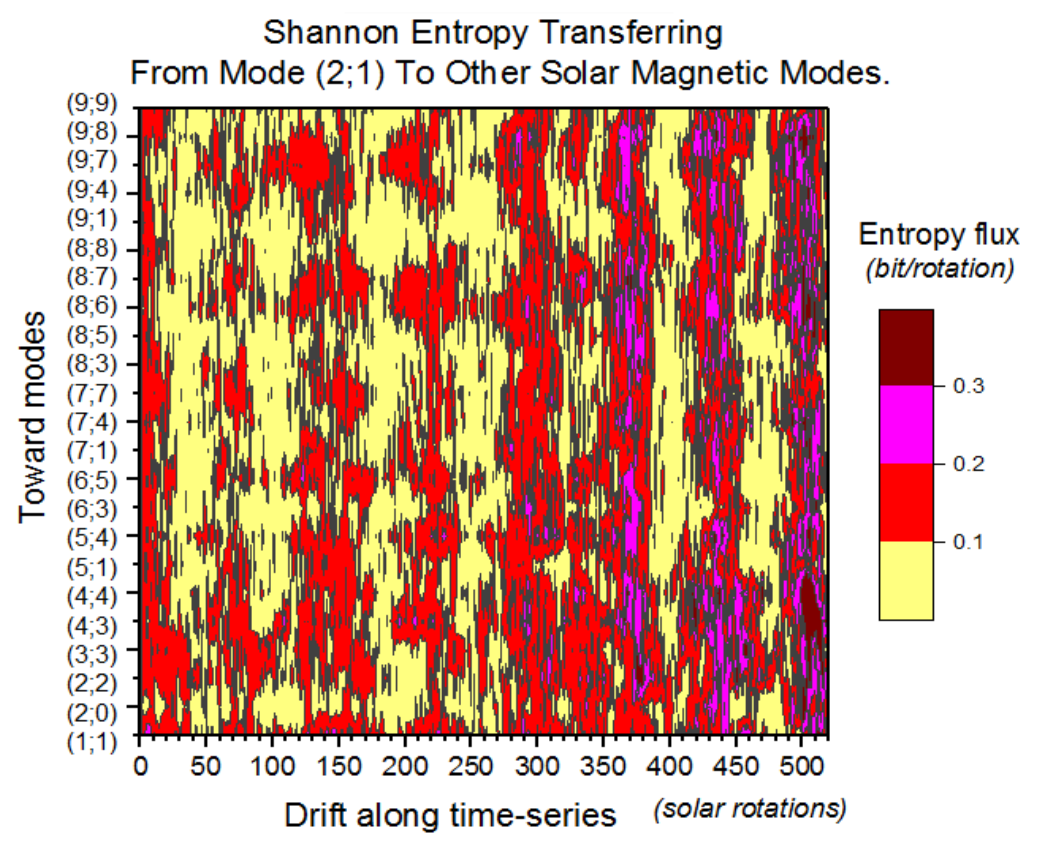

Figure 2. Shannon entropy transfer from $(2 ; 1)$ mode to other modes marked along the vertical axes in the left part of the picture. The horizontal axis shows the drift (in solar rotations) along the timeseries of the mode $(2 ; 1)$. The scale of entropy flux values is shown at the right side of the figure. 
Vertically oriented stripes of red indicate increased values of the information entropy transmitted from mode $(2 ; 1)$. The periodic arrangement of the bands can be seen, in bulk. Below the physical reason of this fundamental feature of the mode $(2 ; 1)$ will be revealed. The increase of modulation amplitudes to the end of the time-series is due to statistical reason when calculating the conditional probabilities in accordance with Formula (2).

Another feature of Shannon's entropy transmission was its circulation. Entropy transfer (from/to) the mode time-series can occur with phase shifts between counter modulated flows. As a result, it leads to a final net entropy flux with a periodic change in direction (flow circulation). Physically, this means that such mode time-series are interdependent. Dependence may be direct or indirect, that does not matter in our case. The sector mode $(8 ; 8)$ has an entropy flow circulation with almost all types of modes. Figure 3 illustrates this finding. The strongest circulation amplitudes appear for tesseral magnetic modes $(l-m=1)$ and modes $(l-m=2),(l-m=4),(l-m=6),(l-$ $m=7)$. Zonal modes $(m=0)$ have no entropy circulation, practically, and are not shown in figure. Figure 3 demonstrates that Shannon entropy circulations for different modes occur with same phase and periodicity (approximately 72 solar rotations). This mysterious situation has not a physical explanation, yet. Almost $70 \%$ of solar magnetic modes have the circulations of informational entropy with mode $(8 ; 8)$. There is no doubt that circulation of informational entropy can be physically interpreted as a manifestation of inter-dependency of magnetic modes. The main role in the network of the interdependence belongs to the sector mode $(8 ; 8)$. Due-to this mode, a cluster of interdependent magnetic modes is organized. This fact plays a very important role in the searching for the physical meaning of the described results.

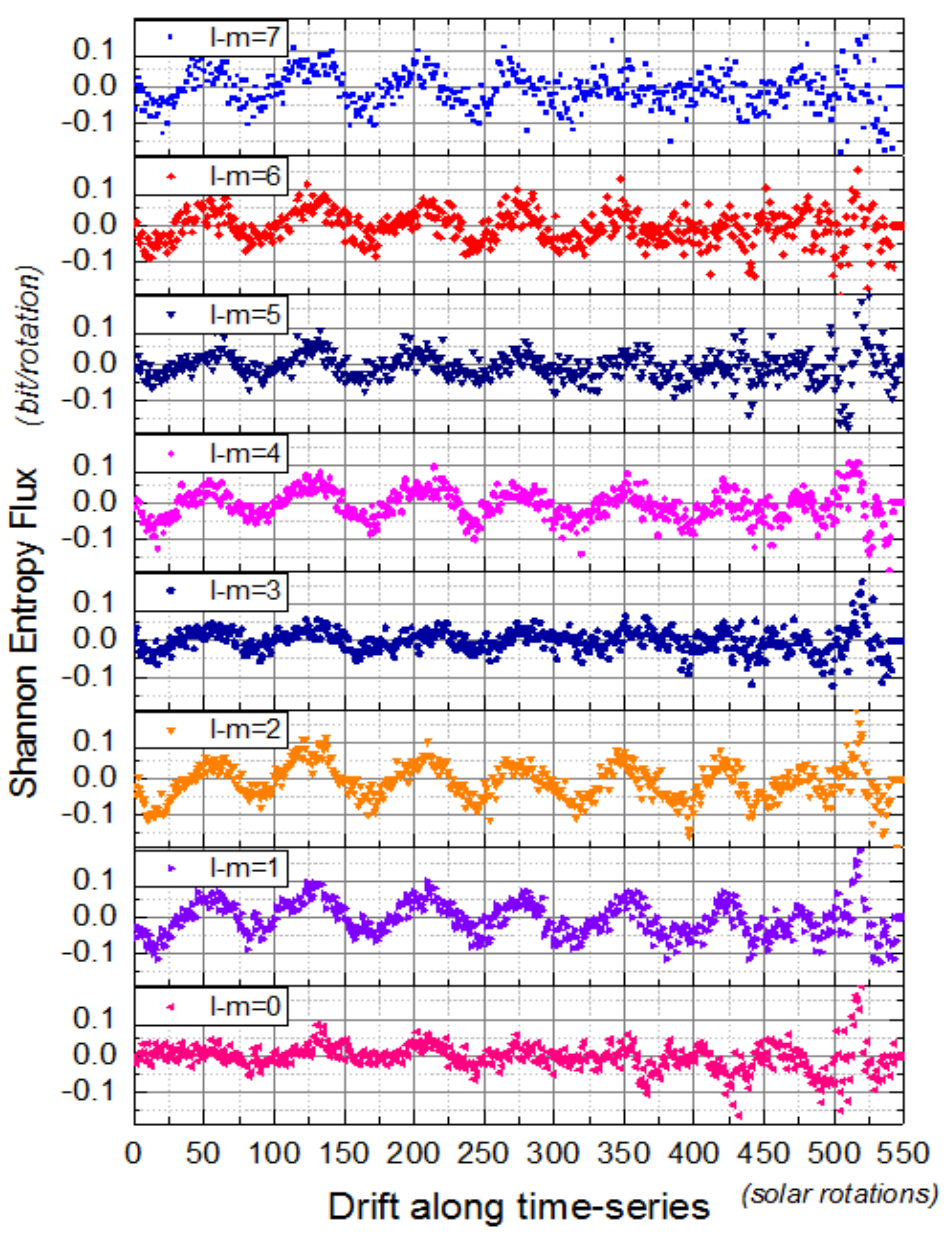

Figure 3. The total circulations of the Shannon entropy between mode $(8 ; 8)$ and modes with different order-degree values: $(l-m)$. 
Analysis of the net informational entropy transferring has given next result. The even zonal modes $(8 ; 0),(6 ; 0),(4 ; 0)$ are entropy "sources" (mean values of net fluxes were mainly positive for these modes), but odd zonal modes $(1 ; 0),(3 ; 0)$ are entropy "absorbents" or entropy "targets". Arrangement of all solar modes according to the averaged net entropy flux values creates sequence of entropy flow over modes. This sequence is illustrated in Figure 4. Three main groups of modes are allocated: the entropy sources, the entropy transmitters, and the entropy targets (or receivers). The entropy sources (modes $(8 ; 0),(6 ; 0)$ and $(4 ; 0))$ have no net entropy flux input; the entropy targets (modes $(1 ; 0)$ and $(3 ; 0)$ ) have no net entropy flux output. The entropy transmitters have inputs and outputs, and are situated between sources and targets. Mode $(2 ; 1)$ is the first in the chain of the entropy transmitters; mode $(9 ; 4)$ is the last one.

\section{Solar Magnetic Modes.}

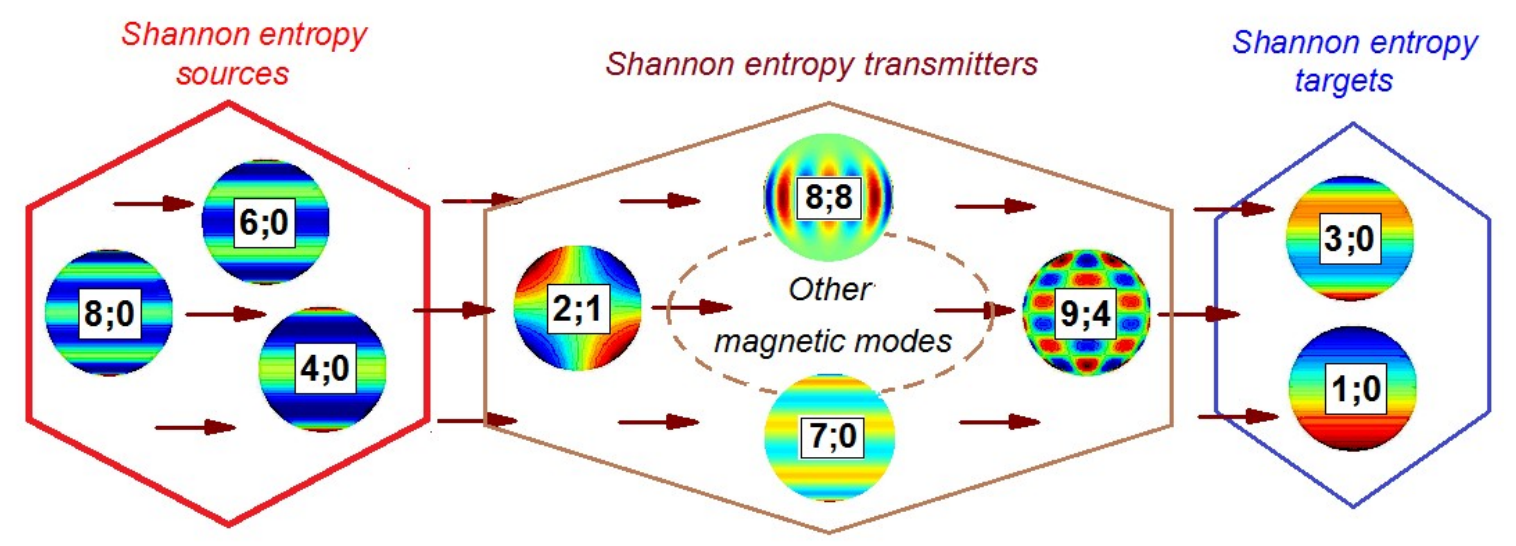

Figure 4. The sequence of the transferring of the net Shannon entropy between solar magnetic modes. Arrows indicate direction of entropy flux. Only magnetic modes with special roles are shown, all others modes are hidden inside the dashed oval. The colors (from blue to red) illustrate mode magnetic polarity structure.

The found main role of even zonal modes as entropy sources is unexpected and should be significant for solar magnetism theory. The domination of tesseral magnetic mode $(2 ; 1)$ in entropy transmitter "family" is also very sudden remarkable fact.

\section{The Special Cluster of Solar Magnetic Modes and a Scenario of It's Behavior}

\subsection{The Physical Meaning of Dependencies between Solar Magnetic Modes}

The process of the Shannon's entropy transferring does not reflect the nature of the source of informational entropy. It is the measure of randomness inherent in the time-series. The value of entropy transfer can shows how much the time-series differ from the Markov chain, the cells of which are filled by random values. This difference, measured in bit/cell, can be interpreted as a degree of influence of "source" time-series to the "target" one. Thus, an entropy transfer amount of $1 \mathrm{bit} / \mathrm{cell}$ (or more) should mean that there are no random values in the target cells of the time-series, because one bit is the total quantum of information. In this regard, for example, entropy flux of 0.1 bit/time can be interpreted as the probability (or share) of nonrandom values in the target timeseries. The influence can be either direct or indirect that doesn't matter in our case. But the existence of dependence is very important to create the physical picture. The modulation of the Shannon entropy transmission over time-series means that there is periodic weakening and strengthening of the dependencies. Physically, this indicates the existence of controlled dynamics. The net Shannon's entropy flux shows in which direction the greatest influence is transmitted. If the informational entropy flow circulates between time-series then they are interconnected. A typical example of the 
Shannon entropy transfer estimation between the time-series of energies of solar magnetic modes is illustrated in Figure 5. The figure shows two-dimensional maps of the Shannon entropy transfer between the sector mode $(8 ; 8)$ and the zonal mode $(8 ; 0)$. The time along the time-series is shown at the horizontal axis, the delay time between the time-series is shown at the vertical axis. Both maps in the figure demonstrate periodic modulation of the informational entropy transfer (green and blue vertical bands). One can see the shift between the modulation bands located at the adjacent maps, which means the circulation of informational entropy between modes.

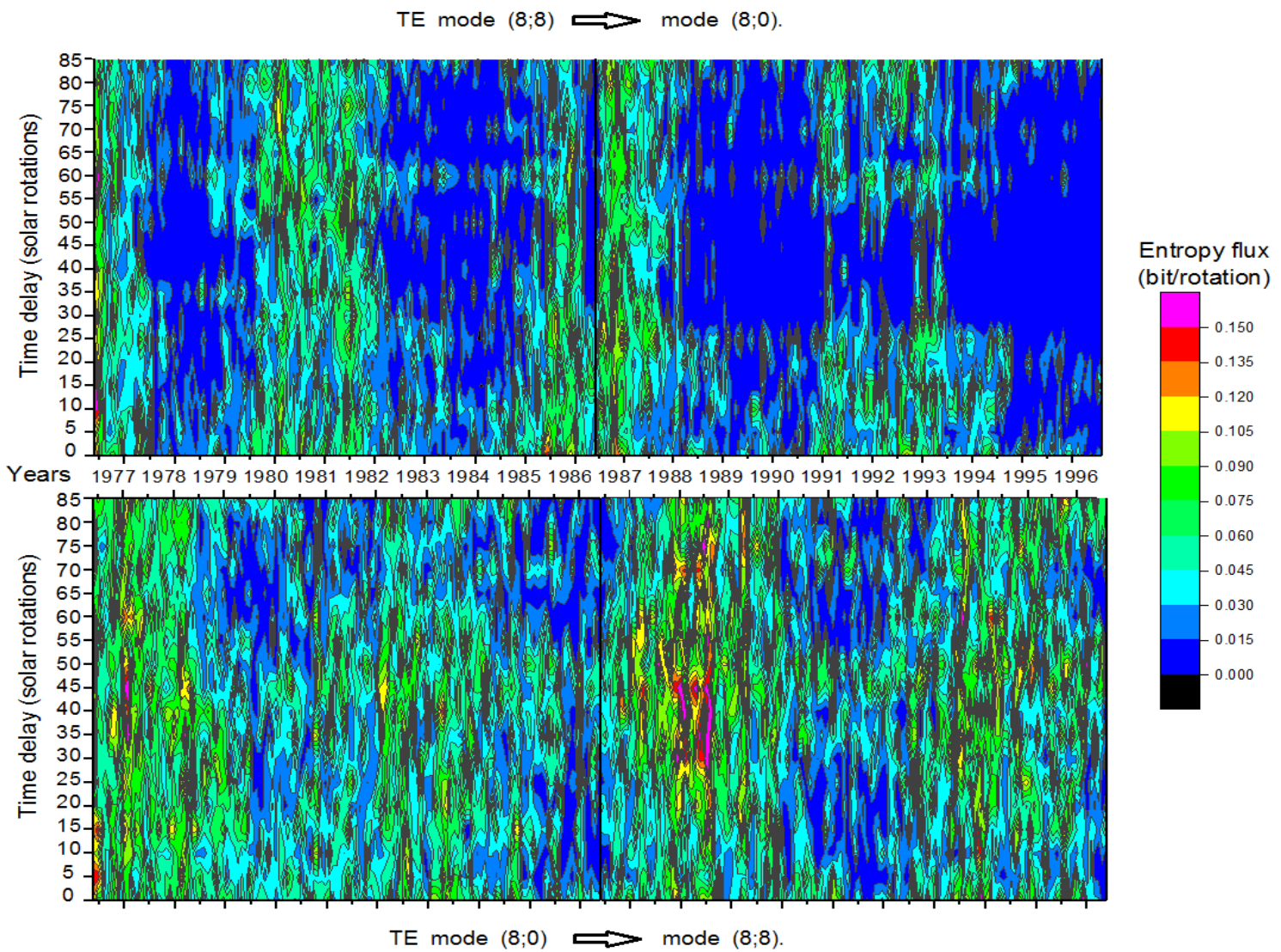

Figure 5. The two-dimensional maps of the Shannon entropy transferring from the mode $(8 ; 8)$ to the mode $(8 ; 0)$ - upper picture, and in back direction - bottom picture. Horizontal axis - is the time in years (from 1976 up to 1997), vertical axis-is the delay-time between the time-series (in solar rotations). The color scale of the entropy flux is shown at the right side of the figure.

The more intense yellow-green colors of the lower map indicate that the amount of information transferred from the mode $(8 ; 0)$ dominates over back transferring from the mode $(8 ; 8)$. All features are preserved in bulk, regardless of the delay-time between the "source" and "target" time-series. This means that the level of dependence between a current state of any cell in the "source" time-series and all future states of the cells in the "target" time-series is maintained for at least 90 solar rotations. This mysterious situation can be understood if we assume that there is some process on the Sun that controls the dynamic system of magnetic modes in accordance with the scenario. Due-to scenario, a periodically repeating cluster of interconnected magnetic modes on the Sun is created (twice per sunspot cycle) as a "template". This cluster could be revealed in the "family" of coupled magnetic modes.

\subsection{The "Template" Composition}

The main intriguing result of the application of the Shannon entropy transfer method in studying of solar magnetic mode interconnection is the discovery of periodic modulation of the 
entropy flux with a period of about 72 solar rotations and the identification of the tesseralquadrupole magnetic mode as the main participant in the processes of modulation of entropy transmission. Thus, the desired "template" should be involved both in modulated entropy transfer from the tesseral-quadrupole mode with a period of 72 solar rotations, and in the "family" of interdependent modes. Such "template" has been found by means of studying the averaged synoptic charts of the magnetic field distribution on the solar surface. The Stanford global magnetic field harmonic coefficients have been used, which have been obtained via the web site http://wso.stanford.edu, courtesy of J.T. Hoeksema. To construct averaged charts we have removed all zonal magnetic modes, because they have not participated in the periodical modulation of entropy. The averaging time-interval from 10 up to 20 solar rotations has been used to reduce noise effects. It should be noted that in the found "template" the large-scale sector modes: $(1 ; 1),(2 ; 1),(3$; $1),(3 ; 2)$ and $(3 ; 3)$ have no played any role practically. Moreover, the removing of these large-scale modes (order $l<4$, degree $m>0$ ) from the cluster of modes has improved the finding. As a result, a periodically appearing polarity structure of the globally tesseral-quadrupole mode has been discovered.

Figure 6 shows how the found tesseral-quadrupole polarity structure correlates with the current intensity of the mode $(2 ; 1)$. It is unexpected, that the cluster of medium- and small-scale modes, without participation of the mode $(2 ; 1)$ has created a tesseral-quadrupole polarity structure periodically. Despite the very low intensity of the mode $(2 ; 1)$, the corresponding polarity structure has appeared (images above the second, fourth, and sixth bars in Figure 6). Obviously, the superposition of the cluster-mode magnetic fields reflects the correspondent structure of the source of the cluster. It is clear that the found cycle does not depend on the sunspot cycles and should have different origin. By means of transferring informational entropy some process periodically is trying create and support the tesseral-quadrupole polarity structure on the Sun.
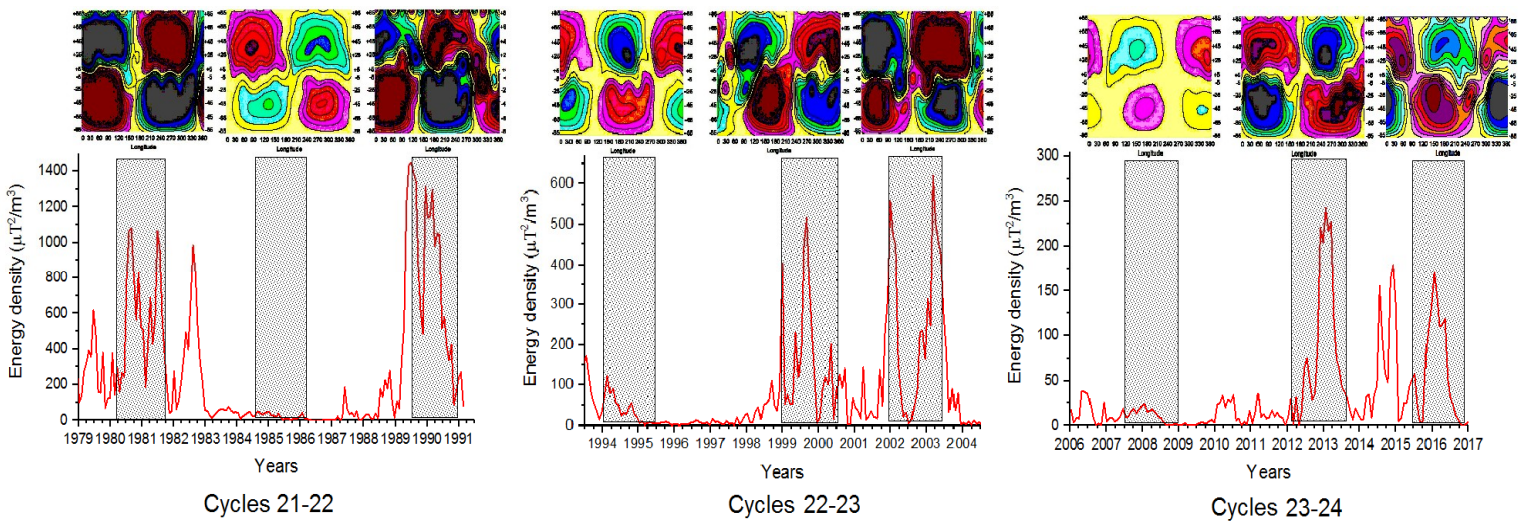

Figure 6. Three graphics of energy density behavior of the mode $(2 ; 1)$ in four solar cycles (red line). At upper part of the figure there are shown the revealed global magnetic field polarity structure in rectangular projection. Red-blue colors in the maps mark different polarities. Vertical bars correspond to the time-intervals, in which the averaging of magnetic field has been made and shown polarity structure has appeared.

\subsection{The Rotation Rate of the Tesseral-Quadrupole Polarity Structure}

The spherical harmonic coefficients are publishing for Carrington longitudes $(T=27.2753$ synodic days). If the uni-polar regions of the tesseral-quadrupole on the solar surface have a different rotation speed, then they will be shifted with time on the synoptic maps in the direction of the solar west (for faster rotation) or in the direction of the solar east (for slower rotation). In Figure 7 , there is shown the time-set of the tesseral-quadrupole polarity configurations. Based on such set, in case of faster rotation one can get the period of $\mathrm{T} \sim 27.0^{\mathrm{d}}$, and in case of slower rotation $-\mathrm{T} \sim 27.5^{\mathrm{d}}$. The faster period is practically coincides with the famous Bartels period which has been detected 
firstly due-to recurrences of the geomagnetic disturbances. We believe that the Bartels period is the true period of rotation of the tesseral-quadrupole polarity structure. Because, there is a simple physical explanation why the period $\sim 13.5^{\mathrm{d}}$ has been frequently recorded in the speed variations of solar wind (Emery et al., 2011). Namely, the global tesseral-quadrupole polarity structure, which periodically appeared on the solar surface, always has two saddle-points near solar equator. These saddle-points have zero magnetic field strength. For such reason, they are open into interplanetary space for high-speed solar winds, as it is for well-known coronal holes. If the rotation of the saddle points occurs with the Bartels period, then frequency of solar wind speed variations near Earth will be as much as twice.

\section{Conclusions}

In this article we have attempted to relate the Shannon entropy transfer results to current state of the knowledge in solar physics and show how the present study advance it's state. Variations of solar magnetic mode intensities do not reveal any stable coordination or communication in changes of magnetic field in global scale. In contrast, the hidden spatial phases, which indicates the position of the field intensity within a given coordinate frame, may be critical in the coordination of global distributed process. This is what has been found in the Sun by using the Shannon entropy transfer technique.

It should be noted that using a derivatives of the modes intensities significantly increases (twothree times) the signal of Shannon entropy transfer. This may serve as indication that in the network of interdependent solar modes the dynamics is more important than the static state. In the discovered regular appearance of the tesseral-quadrupole on the solar surface the most important role have played middle and small-scale magnetic modes $(l>3 ; m>0)$. It can happen only due-to periodic coordination of their spatial distribution. The common known cycle of intensities of solar magnetic fields last approximately 11.2 years. Inside this cycle, the two times shorter cycle of cluster-modes related to their spatial-phases has been revealed. The reason of such kind periodicity in behavior of cluster-modes are still unclear. The unknown process periodically (twice per cycle of sunspots) creates a cluster of interconnected magnetic modes that restore the structure of tesseralqudrupole polarity as a "template". The probable important role of quadrupole-like magnetic fields in common solar cycle formations has been shown theoretically by Popova (2013), Zharkova et al.l. (2017). But authors have considered the cycles of the field intensities. They have found that the quadruple-like field wave arising in the inner layer at the bottom of the solar convection zone explains the occurrence of centennial oscillations of the summary curve of solar activity. Up today, there were no publications dedicated to such hidden solar cycles. We hope that our paper will provoke intense discussion and give to researchers a new approach to understand what it all means. 
The 5th International Electronic Conference on Entropy and Its Applications (ECEA 2019), 18-30 November 2019; Sciforum Electronic Conference Series, Vol. 5, 2019

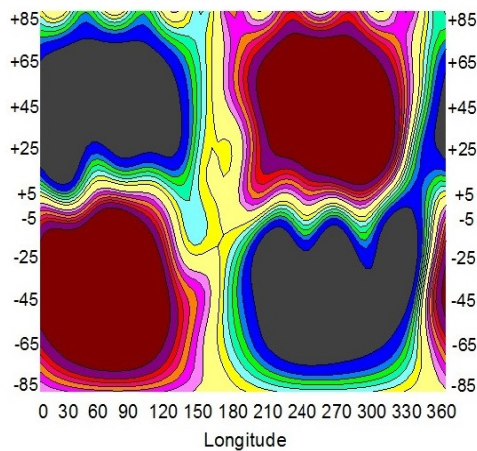

1979.89

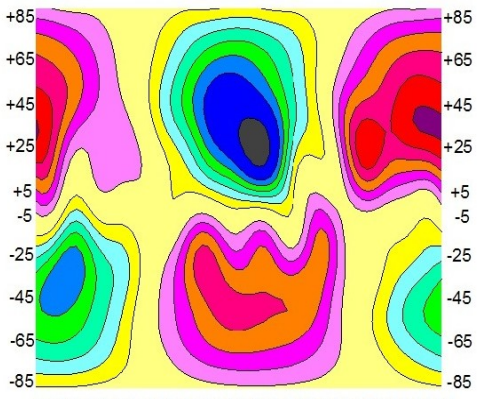

0306090120150180210240270300330360

Longitude

1994.06

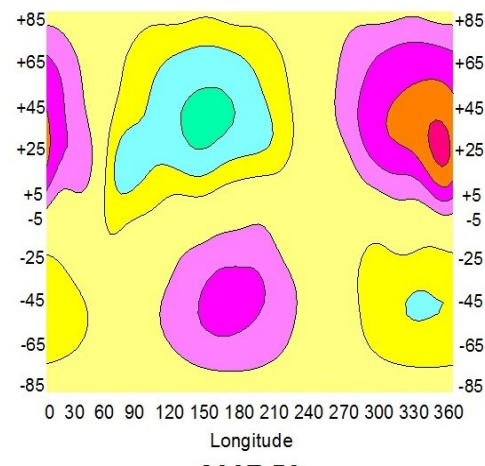

2007.53

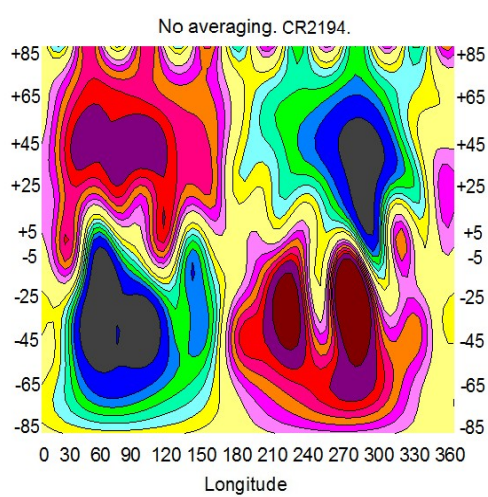

2017.7

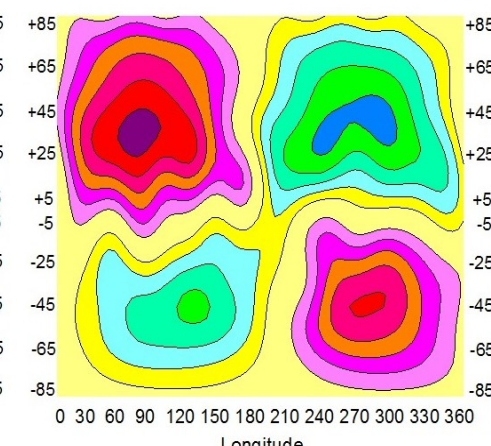

1984.63
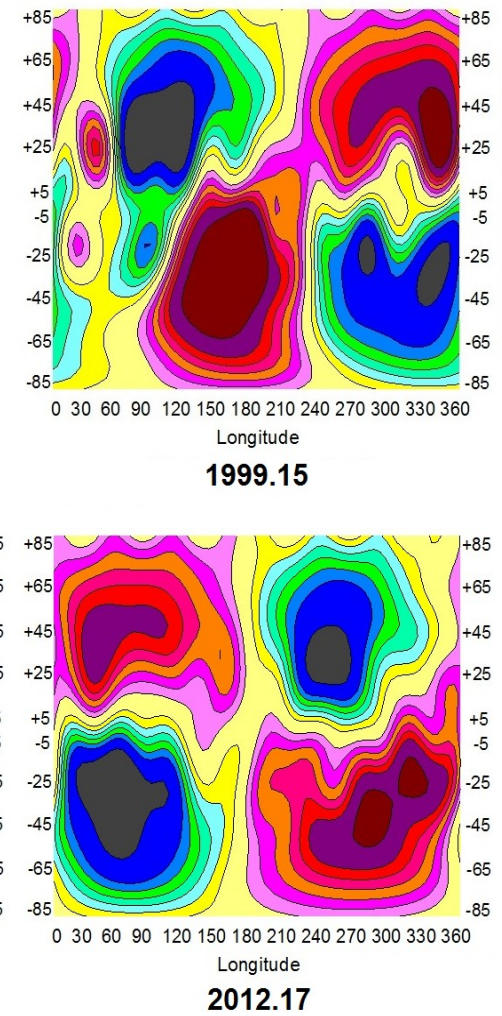

2012.17

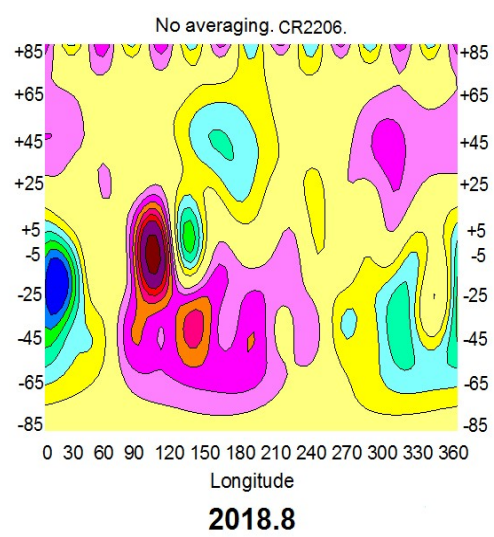

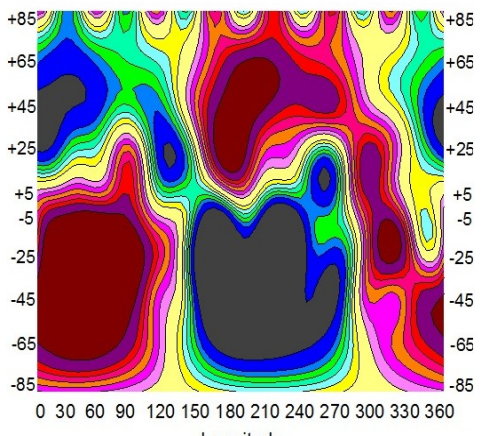

1989.57

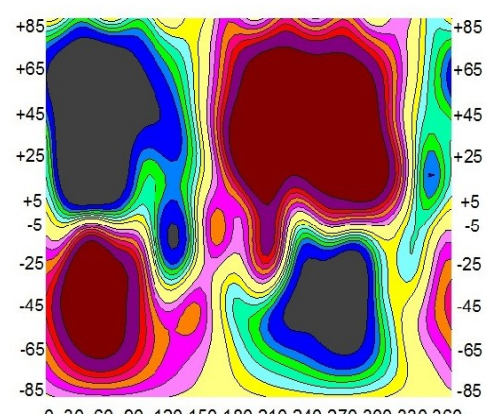

0306090120150180210240270300330360

Longitude

2001.99

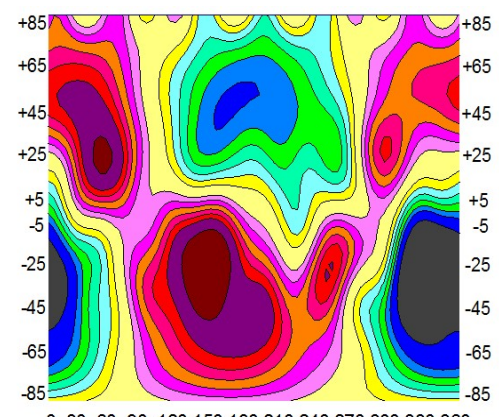

0306090120150180210240270300330360

2015.53

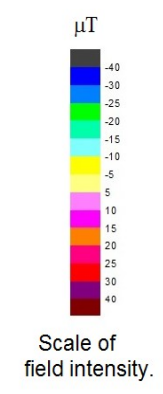

Figure 7. Set of solar tesseral-quadrupole magnetic field configurations. The full solar surface are shown in rectangular projections. The coordinates are solar longitudes and latitudes. Red-blue colors reflects the polarities of magnetic fields. The dates in years are shown at the bottom of every map. The last two maps show the examples of non-averaged tesseral-quadrupole polarity distribution. 
The 5th International Electronic Conference on Entropy and Its Applications (ECEA 2019), 18-30 November 2019; Sciforum Electronic Conference Series, Vol. 5, 2019

\section{References}

1. Emery, B.A.; Richardson, I.G.; Evans, D.S.; Rich, F.J.; Wilson, G.R. Solar Rotational Periodicities and the Semiannual Variation in the Solar Wind, Radiation Belt, and Aurora. Sol. Phys. 2011, 274, 399.

2. James, R.G.; Barnett, N.; Crutchfield, J.P. Santa Fe Institute Working Paper 16-01-001. arXiv 2016, arXiv:1512.06479.

3. Lee, J.; Nemati, S.; Silva, I.; Edwards, B.A.; Butler, J.P.; Malhotra, A. BioMed. Eng. OnLine 2012, 11, 19, doi:10.1186/1475-925X-11-19.

4. Mikhailutsa, V.P. Sol. Phys. 1994, 151, 371.

5. Popova, E.P. A Dynamical System for the Parker Dynamo in the Case of Quadrupole Symmetry of the Magnetic Field. Astronomy Rep. 2013, 57, 310.

6. Schreiber, T. Phys. Rev. Lett. 2000, 85, 461.

7. Zharkova, V.V.; Shepherd, S.J.; Popova, E.; Zharkov, S.I. Reinforcing the double dynamo model with solarterrestrial activity in the past three millennia. arXiv 2017, arXiv:1705.04482v2.

(C) 2019 by the authors. Submitted for possible open access publication under the terms and conditions of the Creative Commons Attribution (CC-BY) license (http://creativecommons.org/licenses/by/4.0/). 